\title{
How many clouds cover the plane?
}

by

James H. Schmerl (Storrs, CT)

Abstract. The plane can be covered by $n+2$ clouds iff $2^{\aleph_{0}} \leq \aleph_{n}$.

According to Komjáth [1], a subset $C$ of the Euclidean plane $\mathbb{R}^{2}$ is a cloud around $\mathbf{a}$ if $\mathbf{a} \in \mathbb{R}^{2}$ and whenever $\ell \subseteq \mathbb{R}^{2}$ is a line and $\mathbf{a} \in \ell$, then $C \cap \ell$ is finite. The following is proved in [1].

Theorem 1 (Komjáth). Suppose that $1 \leq n<\omega$. If $2^{\aleph_{0}} \leq \aleph_{n}$, then $\mathbb{R}^{2}$ can be covered by $n+2$ clouds.

It should be remarked that a somewhat stronger conclusion was proved: whenever $\mathbf{a}_{0}, \mathbf{a}_{1}, \ldots, \mathbf{a}_{n+1} \in \mathbb{R}^{2}$ are $n+2$ distinct noncollinear points, then each $\mathbf{a}_{i}$ has a cloud $C_{i}$ around it such that $\mathbb{R}^{2}=C_{0} \cup C_{1} \cup \ldots \cup C_{n+1}$. A converse to Theorem 1 was proved in [1] for $n=1$. That is, if $\mathbb{R}^{2}$ can be covered by 3 clouds, then the Continuum Hypothesis holds. The converse for $n>1$ was left open. However, Komjáth [2] did prove that it is relatively consistent that $2^{\aleph_{0}}=\aleph_{4}$ and $\mathbb{R}^{2}$ cannot be covered by 3 clouds.

The following converse to Theorem 1 is the main new result in this note.

Theorem 2. Suppose that $1 \leq n<\omega$. If $\mathbb{R}^{2}$ can be covered by $n+2$ clouds, then $2^{\aleph_{0}} \leq \aleph_{n}$.

There are many results closely related to Theorems 1 and 2. Simms [4] presents a thorough historical account of these theorems. We will make use of the following one which Simms attributes to Kuratowski [3].

Theorem 3 (Kuratowski). Suppose that $n<\omega$ and that $X$ is any set. Then $|X| \leq \aleph_{n}$ iff there are $D_{0}, D_{1}, \ldots, D_{n+1} \subseteq X^{n+2}$ which cover $X^{n+2}$ such that $D_{i} \cap \ell$ is finite whenever $i \leq n+1$ and $\ell \subseteq X^{n+2}$ is a line parallel to the ith coordinate axis.

In this theorem we have referred to $\ell$ as a line parallel to the $i$ th coordinate axis; such an $\ell$ is a set for which

2000 Mathematics Subject Classification: 03E50, 51M05. 


$$
\ell=\left\{\left(a_{0}, a_{1}, \ldots, a_{i-1}, x, a_{i+1}, \ldots, a_{n}, a_{n+1}\right) \in X^{n+2}: x \in X\right\}
$$

for some $a_{0}, a_{1}, \ldots, a_{i-1}, a_{i+1}, \ldots, a_{n+1} \in X$.

Proof $\left({ }^{1}\right)$ of Theorem 2. Suppose that $C_{0}, C_{1}, \ldots, C_{n+1}$ cover $\mathbb{R}^{2}$, where each $C_{i}$ is a cloud around $\mathbf{a}_{i}$. Without loss of generality, we assume that each $\mathbf{a}_{i} \neq \mathbf{0}$. We will show that $2^{\aleph_{0}} \leq \aleph_{n}$.

For $2 \leq m<\omega$, consider $\mathbb{R}^{m}$ as a vector space over $\mathbb{R}$, and let $\mathbf{P}_{m}(\mathbb{R})$ be real projective $m$-space, with $\mathbb{R}^{m}$ being embedded in $\mathbf{P}_{m}(\mathbb{R})$ by identifying the point $\left(x_{0}, x_{1}, \ldots, x_{m-1}\right) \in \mathbb{R}^{m}$ with the point in $\mathbf{P}_{m}(\mathbb{R})$ having homogeneous coordinates $\left[x_{0}, x_{1}, \ldots, x_{m-1}, 1\right]$.

For $i<n+2$, let $\infty_{i} \in \mathbf{P}_{n+2}(\mathbb{R})$ be the point at infinity on (every line parallel to) the $i$ th coordinate axis of $\mathbb{R}^{n+2}$, and let $\mathbf{e}_{i} \in \mathbb{R}^{n+2}$ be the $i$ th standard basis vector. Let $S: \mathbf{P}_{n+2}(\mathbb{R}) \rightarrow \mathbf{P}_{n+2}(\mathbb{R})$ be a collineation for which $S\left(\infty_{i}\right)=\mathbf{e}_{i}$ and $S(\mathbf{0})=\mathbf{0}$, and let $T: \mathbb{R}^{n+2} \rightarrow \mathbb{R}^{2}$ be the unique linear transformation such that $T\left(\mathbf{e}_{i}\right)=\mathbf{a}_{i}$. If $\mathbf{x} \in \mathbb{R}^{n+2}$, then $T S(\mathbf{x})$ is defined as long as $\mathbf{x}$ is not in the hyperplane $H \subseteq \mathbb{R}^{n+2}$. Since $\mathbf{0} \notin H$, let $I=(-\varepsilon, \varepsilon)$ be an open interval such that $I^{n+2} \cap H=\emptyset$, and then let $D_{i}=(T S)^{-1}\left(C_{i}\right) \cap I^{n+2}$ for each $i<n+2$. Clearly, the $D_{i}$ 's cover $I^{n+2}$ since the $C_{i}$ 's cover $\mathbb{R}^{2}$.

Let $\ell$ be a line of $\mathbf{P}_{n+2}(\mathbb{R})$ such that $\infty_{i} \in \ell$ and $\ell$ meets $I^{n+2}$. Since $T\left(\mathbf{e}_{i}\right) \neq \mathbf{0}, T S$ is one-one on $\ell \cap I^{n+2}$, and then, since $T S$ preserves collinearity, there is a unique line $\ell^{\prime}$ of $\mathbb{R}^{2}$ which $T S$ maps $\ell$ into. Then $\mathbf{a}_{i}=T S\left(\infty_{i}\right) \in$ $\ell^{\prime}$, so $D_{i} \cap \ell$ is finite. Kuratowski's theorem now applies, so $2^{\aleph_{0}}=|I| \leq \aleph_{n}$.

Komjáth [1] also defines a set $C \subseteq \mathbb{R}^{2}$ to be a circle around $\mathbf{a}$ if $\mathbf{a} \in \mathbb{R}^{2}$ and every half-line from a meets $C$ at no more than one point. Let us say that a cloud around $\mathbf{a}$ is an $n$-cloud if it meets each line through a at no more than $n$ points. Thus, every 1 -cloud is a circle, and every circle is a 2-cloud.

Komjáth [1] proves that $\mathbb{R}^{2}$ can be covered by countably many circles. In fact, he shows that whenever $\mathbf{a}_{0}, \mathbf{a}_{1}, \ldots$ are countably many noncollinear points, then each $\mathbf{a}_{i}$ has a circle $C_{i}$ around it such that $\mathbb{R}^{2}=C_{0} \cup C_{1} \cup \ldots$ His proof works equally well with 1 -clouds. He conjectures that finitely many circles do not suffice to cover $\mathbb{R}^{2}$, and he remarks that he has proved that this is so when the circles are around distinct points. The following affirms Komjáth's conjecture.

THEOREM 4. Finitely many circles cannot cover $\mathbb{R}^{2}$.

Proof. The proof is practically already in the proof of Theorem 2. For a contradiction, suppose that $C_{0}, C_{1}, \ldots, C_{n+1}$ are finitely many circles,

$\left({ }^{1}\right)$ This presentation of the proof owes much to Jan Mycielski who saw what was really going on with my original proof. 
around $\mathbf{a}_{0}, \mathbf{a}_{1}, \ldots, \mathbf{a}_{n+1}$ respectively, which cover $\mathbb{R}^{2}$. We allow the possibility that not all the $\mathbf{a}_{i}$ are distinct, but we do assume that each $\mathbf{a}_{i} \neq \mathbf{0}$. Proceed just as in the proof of Theorem 2 to obtain $T S: \mathbf{P}_{n+2}(\mathbb{R}) \rightarrow \mathbb{R}^{2}$ and $D_{0}, D_{1}, \ldots, D_{n+1} \subseteq I^{n+2} \subseteq \mathbb{R}^{n+2}$. It is clear that each line which is parallel to the $i$ th coordinate axis meets $D_{i}$ in at most 2 points. We show this leads to a contradiction.

Let $N \subseteq I$ be a set having exactly $k=2 n+5$ elements. For each $i \leq n+1$, there are exactly $k^{n+1}$ lines parallel to the $i$ th coordinate axis which meet $N^{n+2}$; hence, $\left|D_{i} \cap N^{n+2}\right| \leq 2 k^{n+1}$. Therefore, $k^{n+2}=\left|N^{n+2}\right| \leq$ $(n+2) \cdot 2 k^{n+1}$, so that $k \leq 2(n+2)$, which is a contradiction.

The proof of Theorem 1 is very robust, being easily adapted to apply to objects other than clouds. For example, let us say that a subset $C \subseteq \mathbb{R}^{2}$ is a spray around $\mathbf{a}$ if the intersection of $C$ with any circle (in the classical Euclidean sense) centered at $\mathbf{a}$ is finite. Then the following can be proved along the lines of the proof of Theorem 1 .

TheOREM 5. Suppose that $1 \leq n<\omega$. If $2^{\aleph_{0}} \leq \aleph_{n}$, then $\mathbb{R}^{2}$ can be covered by $n+2$ sprays.

We do not know if there is a converse to Theorem 5 in the style of Theorem 2.

\section{References}

[1] P. Komjáth, Three clouds can cover the plane, Ann. Pure Appl. Logic 109 (2001), $71-75$.

[2] - A strongly asymmetric function, preprint.

[3] C. Kuratowski, Sur une caractérisation des alephs, Fund. Math. 38 (1951), 14-17.

[4] J. C. Simms, Sierpiński's Theorem, Simon Stevin 65 (1991), 69-163.

Department of Mathematics

University of Connecticut

Storrs, CT 06269, U.S.A.

E-mail: schmerl@math.uconn.edu

Received 28 May 2002;

in revised form 4 April 2003 